\title{
Trends of Antibacterial Resistance at the National Reference Laboratory in Cameroon: Comparison of the Situation between 2010 and 2017
}

\author{
M. Massongo ${ }^{1},{ }^{1,2}$ L. Ngando, ${ }^{1,3}$ E. W. Pefura Yone, ${ }^{1,2}$ Ariane NZouankeu, ${ }^{2}$ W. Mbanzouen, ${ }^{3}$ \\ M. C. Fonkoua, ${ }^{4}$ A. Ngandjio, ${ }^{4}$ J. Tchatchueng, ${ }^{4}$ D. Barger, ${ }^{5}$ and M. C. Tejiokem ${ }^{4}$ \\ ${ }^{1}$ Faculty of Medicine and Biomedical Sciences, University of Yaoundé 1, Yaoundé, Cameroon \\ ${ }^{2}$ Jamot Hospital, Yaoundé, Cameroon \\ ${ }^{3}$ Bacteriology Unit, Cameroon Pasteur Centre, Member of Institut Pasteur International Network, Yaoundé, Cameroon \\ ${ }^{4}$ Epidemiology and Public Health Unit, Cameroon Pasteur Centre, Member of Institut Pasteur International Network, \\ Yaoundé, Cameroon \\ ${ }^{5}$ Bordeaux School of Public Health (ISPED), University of Bordeaux, Inserm 1219 Bordeaux Population Health, Bordeaux, France
}

Correspondence should be addressed to M. Massongo; massongo.massongo@fmsb-uyl.cm

Received 24 March 2021; Revised 28 April 2021; Accepted 11 May 2021; Published 24 May 2021

Academic Editor: Khalid Mehmood

Copyright (C) 2021 M. Massongo et al. This is an open access article distributed under the Creative Commons Attribution License, which permits unrestricted use, distribution, and reproduction in any medium, provided the original work is properly cited.

\begin{abstract}
Introduction. Antimicrobial resistance represents a growing public health threat. One of the World Health Organization's strategic objectives is "strengthening knowledge through surveillance and research." Sub-Saharan African countries are still far from achieving this objective. We aimed to estimate and compare the prevalence of antibacterial resistance in 2010 and 2017 in Cameroon. Methods. We conducted a retrospective study on all clinical specimens cultured in Centre Pasteur du Cameroun (CPC) in 2010 and 2017. Data were extracted from the CPC's laboratory data information system software and then managed and analyzed using R. Bacterial resistance rates were calculated in each year and compared using chi-square or Fisher's tests, and relative changes were calculated. Outcomes included acquired resistance (AR), WHO priority resistant pathogens, some specific resistances of clinical interest, and resistance patterns (multi, extensively, and pan drug resistances) for five selected pathogens. Results. A total of 10,218 isolates were analyzed. The overall AR rate was 96.0\% (95\% CI: 95.4-96.6). Most of WHO priority bacterial resistance rates increased from 2010 to 2017. The most marked increases expressed as relative changes concerned imipenem-resistant Acinetobacter (6.2\% vs. 21.6\%, $+248.4 \%, p=0.02)$, imipenem-resistant Pseudomonas aeruginosa $(13.5 \%$ vs. $23.5 \%,+74.1 \%, p<0.01)$, 3rd generation-resistant Enterobacteriaceae $\left(23.8 \%\right.$ vs. $\left.40.4 \%,+65.8 \%, p<10^{-15}\right)$, methicillin-resistant Staphylococcus aureus $(27.3 \%$ vs. $46.0 \%,+68.6 \%, p<0.002)$, fluoroquinolone-resistant Salmonella (3.9\% vs. $9.5 \%,+142.9 \%, p=0.03)$, and fluoroquinolone-resistant Enterobacteriaceae $\left(32.6 \%\right.$ vs. $\left.54.0 \%,+65.8 \%, p<10^{-15}\right)$. For selected pathogens, global multidrug resistance was high in 2010 and $2017(74.9 \%$ vs. $78.0 \%+4.1 \%, p=0.01)$, intensively drug resistance rate was $5.8 \%(7.0 \%$ vs. $4.7 \% ; p=0.07)$, and no pan drug resistance has been identified. Conclusion. Bacterial resistance to antibiotics of clinical relevance in Cameroon was high and appeared to increase between 2010 and 2017. There is a need for regular surveillance of antibacterial resistance to inform public health strategies and empirically inform prescription practices.
\end{abstract}

\section{Background}

Antimicrobial resistance (AMR) has increased within the past years, and according to the World Health Organization (WHO), this is a growing threat for public health worldwide that calls for urgent and global measures [1]. Although AMR is a natural phenomenon, human factors have a significant impact on its spread. Such factors can be behavioral (inadequate use of antimicrobials, lack of hygiene), structural (weakness in laboratory supply), or managerial (lack of surveillance, inadequate or lacking control programs, poor regulation) [2]. Inadequate use of antimicrobials is one of the 
major AMR suppliers. This includes antibiotic adds in drinking water and daily feed of food-producing animals [3], as well as the intensive use of growth promoters that can encourage the growth of selected resistant bacteria [4]. The overuse, subtherapeutic, or other misuse of antibiotics in animals has shown a correlation with the occurrence of resistant bacteria in humans. These resistant bacteria range from Escherichia coli to Mycobacterium bovis [5-8]. Some factors are more likely to occur in low income countries, such as limited access to a right diagnosis or illicit drug to sell and use, among others [9]. Unregulated antimicrobial use and nonestimation of annual usage are also frequent in developing countries, as shown by Rahman in Pakistan [10].

Facing this growing threat, the WHO published in 2014 a global report on antimicrobial resistance surveillance [11]. Based on national, regional, and global data, this report revealed the sanitary and economic impact of AMR, which included among others: a twofold increase of all-cause mortality rate, an increased risk of infection after admission in intensive care unit, an increased hospitalization stay, an increased frequency of septic shock, and a significant increase of management cost. For many sub-Saharan African (SSA) countries including Cameroon, the expressions "No information obtained for this report" or "No national data available" occured in this first report. At that time, studies on AMR in Cameroon were scarce and old. They were limited in terms of geographical areas and pathogens. However, they showed an increasing AMR rate between the 1990s and the late 2000s [12-15]. One year later, WHO published a country situation analysis of the response to AMR [2], based on a 2year survey with the national sanitary authorities of state members. Only 8 SSA countries (Africa WHO region) out of $47(17.0 \%)$ provided data for this report, while $127 / 147$ (85.0\%) responded for the other WHO regions. African countries showed a lack of national AMR policies, lack of sensitization programs, and inappropriate use of antimicrobials, among others. There was no data from Cameroon in this second report.

After those 2 baseline documents, WHO published in 2016 its Global Action Plan on antimicrobial resistance, based on 5 strategic objectives. The objective number 2 was "to strengthen the knowledge and evidence base through surveillance and research" [16]. We aimed to provide data on the recent evolution of AMR in Cameroon, pursuing a dual purpose: public health (contributing to the objective number 2 of WHO's Global Action Plan) and clinical (possible implications on antibiotics protocols).

\section{Materials and Methods}

2.1. Study Design and Settings. We performed a retrospective study from April 4 to June 1, 2018, in Centre Pasteur du Cameroun (CPC), Yaoundé, the national reference laboratory in biology and public health, and Member of the Pasteur Institute International Network. As a nationwide reference laboratory, CPC analyzes specimen coming from Yaoundé and other regions of the country. We analyzed bacteriological routine data, collected during two distinct one-year periods (2010 and 2017), and stored in the laboratory data informa- tion system (GLIMS). We initially aimed to perform an upto-date ten-year comparison (2007-2017) of the various AMR features targeted, 2017 being the more recent year with completed data. Unfortunately, GLIMS was put in place in July 2009, making 2010 the first year with comprehensive stored data.

2.2. Study Population and Sampling. All bacterial analyses with a valid susceptibility test conducted in CPC in 2010 and 2017 were considered for this study, regardless of their collection site or source of specimens (blood, sputum, urine, stool, pus, etc.). The following were excluded from analysis: samples with isolates from nonpathological swabs (including systematic research with no clinical interest), fungal specimen, or duplicates, especially for the Mycoplasma family, since both Mycoplasma hominis and Ureaplasma urealyticum were systematically reported when one of them was identified (Figure 1).

2.3. Biological Analysis: Procedures and Interpretation. Biological samples had been collected at or transferred to CPC during the enrollment period. For each sample, the name, age, and gender were used to label the corresponding samples and create ID codes for each analysis. Samples were then brought to the bacteriology lab for analysis. Most of the specimen were examined using light microscopy after Gram staining or other appropriate method to assess the presence of pathogens. Blood specimens were incubated at $37^{\circ} \mathrm{C}$ in BactALERT 3D Automaton (Biomerieux) and examined daily for evidence of bacterial growth. When needed, specimens were cultured according to French microbiology guidelines $[17,18]$. Bacteria identification was done using Vitek 2 Compact (Biomerieux) automaton and API kits. The antibiotics susceptibility tests were performed using standard methods (disk, e-test, liquid medium for Vitek 2 from January 2014). Reference documents for these tests were the CASFM (Comité Antibiogramme de la Société Française de Microbiologie) 2010 and 2016 [19, 20]. Results were stored in GLIMS using generated ID codes in tables including patient identification (name, age, gender), sample type or source, microorganism, and susceptibility profile. The later was given through 3 modalities: susceptible (S), intermediate (I), or resistant (R).

2.4. Data Management and Analysis. From GLIMS, we extracted all bacteriology analysis stored in 2010 and 2017 into Excel ${ }^{\circledR}$ tables without patients' name. The crude global database underwent the abovementioned filters (Figure 1). The resulting table was then uploaded to $\mathrm{R}$ version 3.4.3, for data management and analysis. Variables were renamed and harmonized, and new variables were created to fit with our 3 main outcomes:

(i) Global Acquired Resistance. The susceptibility profile was processed to obtain a binary variable (absence or presence of acquired resistance). There was no acquired resistance (wild strain) if a given bacterium was still fully susceptible $(S)$ to all antibiotics (ATB) naturally active in vitro. There was 


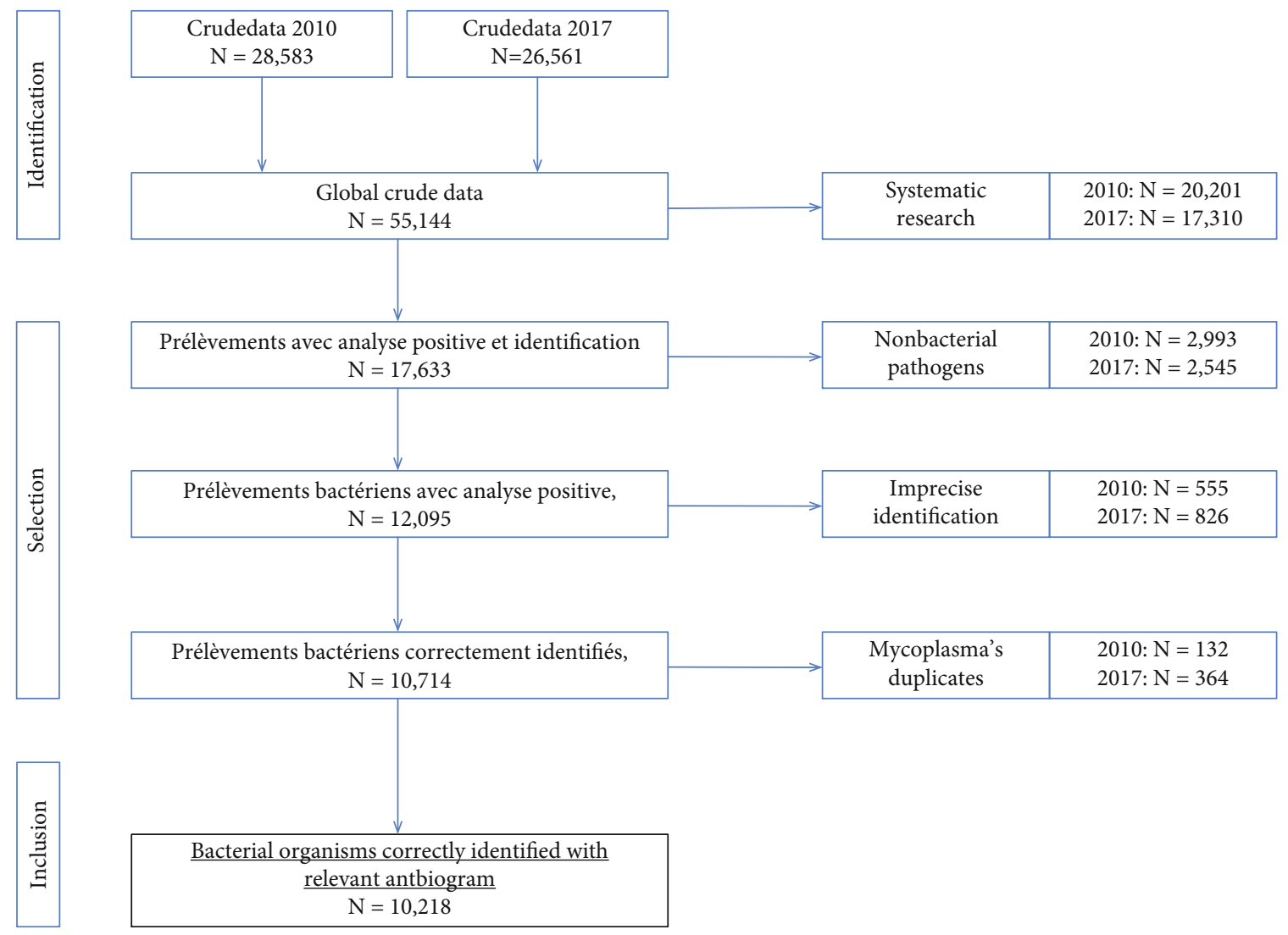

Figure 1: Flow chart of isolate inclusion in the study on antibacterial resistance, Centre Pasteur du Cameroon, 2010 and 2017 , Yaoundé, Cameroon.

acquired resistance (resistant strain) if the pathogen had been found intermediate (I) or resistant (R) for at least one of these ATBs

(ii) Specific Resistance. This informed susceptibility profile of the bacterium regarding a given ATB and permitted identification of WHO's antibiotic-resistant "priority pathogens." These are 12 families of bacteria that pose the greatest threat to human health, divided into three categories according to the urgency of the need for new antibiotics: critical (priority 1), high (priority 2), and medium (priority 3) [21]

(iii) Resistance Pattern. It is applied to Enterobacteriaceae, $P$ aeruginosa, A baumannii, Staphylococcus aureus, and Enterococcus spp, which are frequently involved in healthcare-associated infections. For these bacteria, 5 resistance levels were defined by an international expert proposal from the Centre for Disease Control (CDC) and the European Centre for Disease Control (ECDC): wild, acquired resistant (AR), multidrug-resistant (MDR), extensively drugresistant (XDR), and pan drug-resistant (PDR) bacteria [22]. Wild and resistant bacteria were defined as stated in the variable "susceptibility profile." MDR was defined as acquired nonsusceptibility to at least one agent in three or more antimicrobial categories. XDR was identified when a bacterial isolate remained susceptible to only one or two ATB categories. PDR was defined as nonsusceptibility to all agents in all antimicrobial categories [22]

For each of these outcomes, the corresponding AMR indicators for a given bacterium or group of bacteria were estimated in 2010 and 2017, using the following formula:

Prevalence $=\frac{\text { Number of isolates presenting the given resistance }}{\text { Total number of isolates concerned }} \times 100$.

A relative change (RC) from 2010 to 2017 was then calculated using the following formula:

$$
\mathrm{RC}=\frac{\text { Prevalence in } 2017-\text { Prevalence in } 2010}{\text { Prevalence in } 2010} \times 100
$$

The global prevalence of antibacterial resistance was presented with its $95 \%$ confidence interval. The specific AMR rates were given as numbers and percentages. Betweenperiod comparisons were performed using Chi square or Fisher tests. A $p$ value $<0.05$ was considered as significant.

2.5. Ethical Statements. This work was approved by CPC's administrative board. Since data had been collected routinely prior to the study and not for a research purpose and 
TABle 1: Collection sites distribution, concerning bacterial analysis in 2010 and 2017, Centre Pasteur du Cameroon, Yaounde. Continuous data are expressed in counts \pm standard deviation and categorical data in counts (frequencies in \%).

\begin{tabular}{|c|c|c|c|c|c|}
\hline Variable & Modalities & Overall & 2010 & 2017 & $p$ value \\
\hline \multirow{3}{*}{$\begin{array}{l}\text { Sex* } \\
N=7,588\end{array}$} & Female & $4,748(62.6)$ & $2,274(63.4)$ & $2,474(61.9)$ & 0.187 \\
\hline & Male & $2,840(37.4)$ & $1,315(36.6)$ & $1,525(38.1)$ & \\
\hline & Total & $7,588(100.0)$ & $3,589(100.0)$ & $3,999(100.0)$ & \\
\hline \multirow{4}{*}{$\begin{array}{l}\text { Age }^{*} \\
N=7,711\end{array}$} & Mean & $30.2 \pm 21.0$ & $29.2 \pm 20.0$ & $31.1 \pm 22.0$ & $<0.001$ \\
\hline & Adult ( $>18$ years) & $5,742(74.5)$ & $2,684(74.9)$ & $3,058(74.0)$ & 0.343 \\
\hline & Teen/child $(\leq 18)$ & $1,969(25.5)$ & $897(25.1)$ & $1,072(26.0)$ & \\
\hline & Total & $7,711(100.0)$ & $3,581(100.0)$ & $4,130(100.0)$ & \\
\hline \multirow{5}{*}{$\begin{array}{l}\text { Specimen } \text { group }^{* *} \\
N=8985\end{array}$} & Deep & $4,350(48.4)$ & $2,012(48.1)$ & $2,238(48.7)$ & 0.280 \\
\hline & Female genital & $3,290(36.6)$ & $1,525(36.4)$ & $1,765(36.8)$ & \\
\hline & Male genital & $711(7.9)$ & $332(7.9)$ & $379(7.9)$ & \\
\hline & Superficial & $634(7.1)$ & $319(7.6)$ & $315(6.6)$ & \\
\hline & Total & $8,985(100.0)$ & $4,188(100.0)$ & $4,697(100.0)$ & \\
\hline
\end{tabular}

*Sex and age were those informed on each collection site. Given the possibility for a patient to provide more than one sample, there were more samples than patients. ${ }^{* *}$ Deep specimen included blood cultures, urine, stool, foreign bodies, soft tissue, broncho-pulmonary, bone-and-joints, pleural effusion, cerebrospinal fluid, intraperitoneal, liver punctures, cerebral, lymph nodes, and gastric liquid. Superficial ones were skin, throat, nasal or wound swab, and any externalized fluid.

personal contact details were not available, individual consents could not be obtained from participants. Data were analyzed anonymously. The study induced no risk for patients or CPC staff.

\section{Results}

3.1. Study Population, Specimens, and Bacterial Isolates. A total of 55,144 analysis were extracted; $48.2 \%$ of them were realized from 2017. Analyses resulting from systematic demands or screening purpose (such as beta hemolytic streptococci in throat, Escherichia, Campylobacter, and other entero-pathogens in stool, Candida in stool and vaginal fluid, nasal or throat staphylococci, and vaginal streptococcus B) were first excluded, since they did not reflect a clinical infectious situation, and most of them had negative results (which were systematically reported and stored). These 37,511 analyses accounted for $2 / 3$ of the total. Nonbacterial isolates (mainly fungi) were then excluded, followed by bacterial one for which the identification was imprecise or not completed (such as "Gram-negative bacillus," "Gram-positive bacteria," and "Other staphylococcus"). Lastly, duplicates of Mycoplasmas (Mycoplasma hominis and Ureaplasma urealyticum) were eliminated. These duplicates appeared for some isolates and consisted in a second line for the same patient and the same specimen but without any susceptibility test. This selection process led to a final sample of 10,218 isolates (Figure 1).

These isolates originated from 7,314 patients, 3,394 (46.4\%) enrolled in 2010, and 3,920 (53.6\%) in 2017. Their mean age \pm standard deviation was $30.2 \pm 21.0$ years, nearly $75 \%$ of them were adults, and $62.6 \%$ were woman. There were 8,985 distinct specimens, from 23 different collection sites. The collection sites were dominated by female genital (32.7\%) and urinary (28.7\%) ones. These samples were gathered into 4 groups, according to their origin and clinical rel- evance. Comparison between 2010 and 2017 according to patients' gender and age as well as collection sites is shown in Table 1.

A total of 71 bacteria species were identified and grouped into 14 categories, according to morphological and susceptibility criteria (Table 2). Genital Mycoplasma accounted for one-third of the total. Apart from those genital tract commensals, Escherichia coli (17.0\%), Klebsiella pneumoniae (8.2\%), and Staphylococcus aureus (5.0\%) were the 3 more frequent bacteria identified in this study.

3.2. Global Acquired Resistance. Of the 10,218 bacterial isolates, 5,405 (52.8\%) underwent a proper acquired resistance estimation. The other were excluded mainly due to missing or discordant data in the susceptibility tests $(45.9 \%)$ and in a few cases for insignificant number (1.3\%).

Among these 5,405 isolates, 5,189 were resistant to at least one antibiotic usually active in vitro, giving an overall prevalence $(95 \% \mathrm{CI})$ of acquired resistance in this bacterial population of $96.0 \%$ (95.4-96.6)\%. This prevalence increased from 2010 to 2017 ( $92.8 \%$ to $99.2 \%, \mathrm{RC}=+6.9 \%, p<0.0001$ ). Four bacterial groups, accounting for $75.3 \%$ of the total, had a $100 \%$ acquired resistance rate in 2010 and 2017. These were Enterobacteriaceae, other fermenting GNB, Enterococci, and Anaerobic (Table 3). In addition, Streptococcus pneumoniae (56 isolates), Pseudomonas aeruginosa (195 isolates), and Acinetobacter spp (150 isolates) also had a 100\% resistance rate at the two data collecting periods.

3.3. Specific Resistances. Globally, an increase in WHO priority resistances was observed between 2010 and 2017, with relative changes ranging from $+32.4 \%$ (Ampicillin- $\mathrm{R}$ Haemophilus influenzae) to $+248.4 \%$ (Imipenem-R Acinetobacter baumannii). Only imipenem-resistant Enterobacteriaceae were less frequent in 2017 than 2010 (RC of -10.8\%). For an unknown reason, fluoroquinolones (FQ) susceptibility was not tested 
TABLe 2: Bacterial groups identified in 2010 and 2017 in Centre Pasteur du Cameroun.

\begin{tabular}{lccc}
\hline Bacterial group & Isolates $2010(\%)$ & Isolates 2017 (\%) & Over all (\%) \\
\hline Genital Mycoplamas & $1,637(34.8)$ & $1,852(33.6)$ & $3,489(34.1)$ \\
Group 1 Enterobacteriaceae & $1,162(24.7)$ & $1,057(19.2)$ & $2,219(21.7)$ \\
Staphylococci & $375(8.0)$ & $669(12.1)$ & $1,044(10.2)$ \\
Group 2 Enterobacteriaceae & $456(9.7)$ & $469(8.5)$ & $925(9.1)$ \\
Streptococci & $319(6.8)$ & $321(5.8)$ & $640(6.3)$ \\
Group 3 Enterobacteriaceae & $225(4.8)$ & $301(5.5)$ & $526(5.1)$ \\
Nonfermenting Gram-negative bacilli & $221(4.7)$ & $273(5.0)$ & $494(4.8)$ \\
Enterococci & $126(2.7)$ & $287(5.2)$ & $413(4.0)$ \\
Other Gram-negative bacilli & $63(1.3)$ & $110(2.0)$ & $134(1.3)$ \\
Gram-negative cocci & $22(0.5)$ & $44(0.8)$ & $132(1.3)$ \\
Other Gram-positive cocci & $23(0.5)$ & $0(0.0)$ & $67(0.7)$ \\
Vibrio cholerae & $58(1.2)$ & $36(0.7)$ & $58(0.6)$ \\
Gram-positive bacilli & $3(0.1)$ & $23(0.4)$ & $39(0.4)$ \\
Anaerobic & $15(0.3)$ & $5,513(100.0)$ & $38(0.4)$ \\
Total & $4,705(100.0)$ & $10,218(100.0)$ \\
\hline
\end{tabular}

Enterobacteriaceae resistance groups: Group 1 = no beta - lactamase, Group 2 = low - level penicillinase, Group 3 = low - level cephalosporinase.

TABle 3: Acquired bacterial resistance (AR) and relative change between 2010 and 2017 according to bacterial groups, Centre Pasteur du Cameroon, Yaoundé.

\begin{tabular}{|c|c|c|c|c|c|c|c|c|}
\hline \multirow{2}{*}{ Bacterial group } & \multicolumn{2}{|c|}{ Overall } & \multicolumn{2}{|c|}{2010} & \multicolumn{2}{|c|}{2017} & \multirow{2}{*}{ Relative change } & \multirow{2}{*}{$p$ value } \\
\hline & Isolates & $\operatorname{AR}(\%)$ & Isolates & $\operatorname{AR}(\%)$ & Isolates & $\operatorname{AR}(\%)$ & & \\
\hline Enterobacteriacae & 3,371 & $3,371(100.0)$ & 1,684 & $1,684(100.0)$ & 1,687 & $1,687(100.0)$ & 0.0 & - \\
\hline Nonfermenting GNB* & 392 & $380(96.9)$ & 167 & $162(97.0)$ & 225 & $218(96.9)$ & -0.1 & 0.30 \\
\hline Other GNB & 113 & $113(100.0)$ & 54 & $54(100.0)$ & 59 & $59(100.0)$ & 0.0 & - \\
\hline Staphylococci & 419 & $330(78.8)$ & 266 & $183(88.8)$ & 153 & $147(96.1)$ & +39.7 & $<0.001$ \\
\hline Streptococci & 505 & $391(77.4)$ & 300 & $195(65.0)$ & 205 & $196(95.6)$ & +47.1 & $<0.001$ \\
\hline Enterococci & 408 & $408(100.0)$ & 126 & $126(100.0)$ & 282 & $282(100.0)$ & 0.0 & - \\
\hline Gram-negative cocci & 124 & $123(99.2)$ & 15 & $15(100.0)$ & 109 & $108(99.1)$ & -0.9 & 1 \\
\hline Anaerobic & 17 & $17(100.0)$ & 9 & $9(100.0)$ & 8 & $8(100.0)$ & 0.0 & - \\
\hline Vibrio cholerae & 56 & $56(100.0)$ & 56 & $56(100.0)$ & 0 & $0(0.0)$ & - & 1 \\
\hline Total & 5,405 & $5,189(96.0)$ & 2,677 & $2,484(92.8)$ & 2,728 & 2,705 (99.2) & +6.9 & $<0.001$ \\
\hline
\end{tabular}

${ }^{*}$ GNB: Gram-negative bacilli.

for Neisseria gonorrhoeae in 2010, but only in 2017. FQ-R $N$ gonorrhoeae had the highest rate $(80.9 \%)$ among the 12 WHO priority pathogens in 2017. Three specific resistances not observed in 2010 were present in 2017: Vancomycin-resistant Enterococcus spp, 3rd generation cephalosporin- (3GC-) resistant Neisseria gonorrhoeae, and fluoroquinolone-resistant Shigella spp. Concerning other pathogens and resistances, Mycoplasma resistance was high and slightly increased for fluoroquinolones, while it decreased for cyclins, between 2010 and 2017. There was a >100\% increase in 3GC-resistant Escherichia coli (Table 4).

3.4. Resistance Patterns. As mentioned in Material and Methods, resistance pattern determination applied to Enterobacteriaceae, $P$ aeruginosa, A baumannii, Staphylococcus aureus, and Enterococcus spp. Overall, 4,903 (47.9\%) strains were eligible. Among these, 4,474 fulfilled the required criteria, on which $3,422(76.5 \%)$ were classified multidrug resistance (MDR). Global resistance patterns and their comparison between 2010 and 2017 are shown in Table 5 .

No pan drug resistance (PDR) was identified. The between-period comparison differed from one bacterium to another. MDR increased distinctly for $S$. aureus, while it tended to remain stable for the 4 other pathogens. Conversely, extensively drug resistance (XDR) increased very highly for A. baumannii and decreased for the other pathogens, although most of the differences were not statistically significant (Table 6).

\section{Discussion}

More than 10,000 antibiotic susceptibility tests were analyzed in this study. Genital mycoplasmas were the most frequent bacteria, followed by E. coli. Swabs were clinically relevant for $>90 \%$ of them. Almost $100 \%$ of strains had an acquired resistance at baseline. All WHO priority resistances highly 
TABLE 4: Some specific resistances of clinical interest as per WHO priority resistances and their relative change between 2010 and 2017 , Centre Pasteur du Cameroun, Yaounde.

\begin{tabular}{|c|c|c|c|c|c|c|c|c|c|}
\hline Categories & Resistance features & Isolates & $\begin{array}{l}\text { verall } \\
\text { Feature } \\
\text { frequency } \\
(\%)\end{array}$ & Isolates & $\begin{array}{l}010 \\
\text { Feature } \\
\text { frequency } \\
(\%)\end{array}$ & Isolates & $\begin{array}{l}017 \\
\text { Feature } \\
\text { frequency } \\
(\%)\end{array}$ & $\begin{array}{c}\text { Relative } \\
\text { change } \\
(\%)\end{array}$ & $p$ value \\
\hline \multirow{4}{*}{ WHO priority 1} & $\begin{array}{c}\text { Imipenem- } \mathrm{R}^{1} \text { Acinetobacter } \\
\text { baumannii }\end{array}$ & 167 & 15.6 & 65 & 6.2 & 102 & 21.6 & +248.4 & 0.032 \\
\hline & $\begin{array}{c}\text { Imipenem-R Pseudomonas } \\
\text { aeruginosa }\end{array}$ & 234 & 18.4 & 119 & 13.5 & 115 & 23.5 & +74.1 & $<0.01$ \\
\hline & Imipenem-R Enterobacteriaceae & 2,066 & 3.5 & 504 & 4.0 & 1,562 & 3.3 & -17.5 & $<10^{-8}$ \\
\hline & $3 \mathrm{GC}^{2}-\mathrm{R}$ Enterobacteriaceae & 3,643 & 32.1 & 1,818 & 23.8 & 1,825 & 40.4 & +69.8 & $<10^{-15}$ \\
\hline \multirow{7}{*}{ WHO priority 2} & Vancomycin-R Enterococcus spp & 412 & 0.5 & 126 & 0.0 & 284 & 0.7 & - & 1 \\
\hline & Methicillin-R Staphylococcus aureus & 412 & 34.2 & 262 & 27.3 & 150 & 46.0 & +68.6 & $<10^{-3}$ \\
\hline & $\begin{array}{l}\text { Fluoroquinolone-R } \\
\text { Campylobacter spp }\end{array}$ & 19 & 52.6 & 12 & 41.7 & 7 & 71.4 & +71.4 & 0.3 \\
\hline & Fluoroquinolone-R Salmonella spp & 144 & 5.6 & 103 & 3.9 & 41 & 9.5 & +142.9 & 0.03 \\
\hline & 3GC-R Neisseria gonorrhoeae & 123 & 1.6 & 18 & 0.0 & 105 & 1.9 & - & 1 \\
\hline & Quinolone-R Neisseria gonorrhoeae & 18 & 16.7 & 18 & 16.7 & 0 & - & - & - \\
\hline & $\begin{array}{l}\text { Fluoroquinolone-R Neisseria } \\
\text { gonorrhoeae }\end{array}$ & 105 & 80.9 & 0 & - & 105 & 80.9 & - & - \\
\hline \multirow{3}{*}{ WHO priority 3} & $\begin{array}{l}\text { Penicillin nonsusceptible } S^{3} \text {. } \\
\text { pneumoniae }\end{array}$ & 84 & 5.9 & 56 & 5.2 & 28 & 7.6 & +47.0 & 0.09 \\
\hline & $\begin{array}{c}\text { Ampicillin-R Haemophilus } \\
\text { influenzae }\end{array}$ & 62 & 70.9 & 9 & 55.6 & 53 & 73.6 & +32.4 & $<0.01$ \\
\hline & Fluoroquinolone-R Shigella $s p p$ & 128 & 0.8 & 53 & 0.0 & 75 & 2.7 & - & 1 \\
\hline \multirow{7}{*}{$\begin{array}{l}\text { Other specific } \\
\text { resistances }\end{array}$} & $\begin{array}{l}\text { Fluoroquinolone- } \mathrm{R} \\
\text { Enterobacteriaceae }\end{array}$ & 3,447 & 45.1 & 1710 & 34.0 & 1737 & 56.0 & +65.8 & $<10^{-15}$ \\
\hline & 3GC-R Escherichia coli & 1,720 & 25.9 & 880 & 17.1 & 840 & 35.2 & +105.8 & $<10^{-15}$ \\
\hline & Fluoroquinolone-R Escherichia coli & 1,693 & 49.9 & 867 & 37.4 & 826 & 61.6 & +64.5 & $<10^{-15}$ \\
\hline & Fluoroquinolone-R Mycoplasmas & 3,505 & 63.8 & 1,636 & 62.8 & 1,869 & 64.6 & +1.8 & $<10^{-3}$ \\
\hline & Fluoroquinolone-I/R ${ }^{4}$ Mycoplasmas & 3,505 & 97.6 & 1,636 & 98.6 & 1,869 & 96.8 & -1.8 & $<10^{-3}$ \\
\hline & Cyclin-R Mycoplasmas & 3,505 & 27.2 & 1,636 & 32.7 & 1,869 & 22.3 & -31.8 & $<10^{-13}$ \\
\hline & Josamycin-R Mycoplasmas & 3,505 & 1.10 & 1,636 & 1.1 & 1,869 & 1.2 & +0.1 & 0.90 \\
\hline
\end{tabular}

TABLE 5: Overall resistance pattern rates on five selected pathogens and their comparison between 2010 and 2017.

\begin{tabular}{lcccccccc}
\hline \multirow{2}{*}{ Resistance pattern } & \multicolumn{2}{c}{ Overall } & \multicolumn{2}{c}{2010} & \multicolumn{2}{c}{2017} & \multirow{2}{*}{ Relative change } & $p$ value \\
& Numbers & Rate, $\%$ & Numbers & Rate, \% & Numbers & Rate, \% & 1.00 \\
\hline Wild & 5 & 0.1 & 2 & 0.1 & 3 & 0.1 & 29.5 & -5.4 \\
Acquired resistance & 787 & 17.6 & 393 & 18.1 & 394 & 17.1 & 0.43 \\
Multidrug resistance & 3422 & 76.5 & 1628 & 74.9 & 1794 & 78.0 & 4.1 & 0.01 \\
Extensively drug resistance & 260 & 5.8 & 152 & 7.0 & 108 & 4.7 & -48.8 & $<0.01$ \\
Total & 4474 & 100.0 & 2175 & 100.0 & 2299 & 100.0 & 0.0 \\
\hline
\end{tabular}

increased between 2010 and 2017 except Imipenem-R Enterobacteriaceae which slightly decreased. Three resistances not seen in 2010 were present 2017 in this category (Vancomycin-R Enterococcus spp, 3GC-R Neisseria gonorrhoeae, and Fluoroquinolone-R Shigella spp). The prevalence of penicillin nonsusceptible $S$. pneumoniae was low but increased between the 2 periods, while $>50 \%$ of $H$. influenzae were initially ampicillin-R. FQ-R Enterobacteriaceae were frequent in 2010 and almost doubled 7 years later. Less than $5 \%$ of genital mycoplasmas were still fully susceptible to FQ, while the susceptibility to josamycine remained active for nearly all of them. More than $75 \%$ of eligible strains were MDR, and no PDR was identified.

Our sample size allowed relevant counts for most of bacteria of clinical interest. This size was higher than most of those we found in other Cameroonian or African studies 
TABle 6: Detailed resistance patterns per bacteria and their comparison between 2010 and 2017, Centre Pasteur du Cameroon, Yaoundé, Cameroon.

\begin{tabular}{|c|c|c|c|c|c|c|}
\hline \multirow{2}{*}{ Bacteria } & \multirow{2}{*}{ Resistance pattern } & \multicolumn{3}{|c|}{ Numbers (\%) } & \multirow{2}{*}{ Relative change } & \multirow{2}{*}{$p$ value } \\
\hline & & Overall & 2010 & 2017 & & \\
\hline \multirow{4}{*}{ Enterobacteriaceae } & $\mathrm{AR}$ & $448(13.2)$ & $234(13.7)$ & $214(12.7)$ & $-7.3 \%$ & \\
\hline & MDR & $2,748(81.1)$ & $1,362(79.8)$ & $1,386(82.4)$ & $+3.2 \%$ & 0.09 \\
\hline & $\mathrm{XDR}$ & $193(5.7)$ & $110(6.5)$ & $83(4.9)$ & $-23.4 \%$ & \\
\hline & Total & $3,389(100.0)$ & $1,706(100.0)$ & $1,683(100)$ & - & \\
\hline \multirow{4}{*}{ Pseudomonas aeruginosa } & $\mathrm{AR}$ & $54(27.7)$ & $25(25.9)$ & $29(29.9)$ & $+15.5 \%$ & \\
\hline & MDR & $109(55.9)$ & $53(54.1)$ & $56(57.7)$ & $+6.7 \%$ & 0.33 \\
\hline & $\mathrm{XDR}$ & $32(16.4)$ & $20(20.4)$ & $12(12.4)$ & $-39.2 \%$ & \\
\hline & Total & $195(100.0)$ & $98(100.0)$ & $97(100.0)$ & - & \\
\hline \multirow{4}{*}{ Acinetobacter spp } & $\mathrm{AR}$ & $52(34.7)$ & $21(37.5)$ & $31(33.0)$ & $-10.7 \%$ & \\
\hline & MDR & $84(56.0)$ & $32(57.1)$ & $52(55.3)$ & $-1.8 \%$ & 0.44 \\
\hline & XDR & $14(9.3)$ & $3(5.4)$ & $11(11.7)$ & $+116.7 \%$ & \\
\hline & Total & $150(100.0)$ & $56(100.0)$ & $94(100.0)$ & - & \\
\hline \multirow{4}{*}{ Enterococcus spp } & $\mathrm{AR}$ & $78(19.2)$ & $11(8.7)$ & $67(23.8)$ & $+58.6 \%$ & \\
\hline & MDR & $317(77.9)$ & $104(82.6)$ & $213(75.8)$ & $-8.1 \%$ & $<10^{-7}$ \\
\hline & $\mathrm{XDR}$ & $12(2.9)$ & $11(8.7)$ & $1(0.4)$ & $-95.4 \%$ & \\
\hline & Total & $407(100.0)$ & $126(100.0)$ & $281(100.0)$ & - & \\
\hline \multirow{5}{*}{ Staphylococcus aureus } & Wild & $5(1.6)$ & $2(1.1)$ & $3(2.1)$ & $+90.9 \%$ & \\
\hline & $\mathrm{AR}$ & $155(46.6)$ & $102(54.0)$ & $53(36.8)$ & $-17.2 \%$ & \\
\hline & MDR & $164(49.2)$ & $77(40.7)$ & $87(60.4)$ & $+48.4 \%$ & $<0.001$ \\
\hline & $\mathrm{XDR}$ & $9(2.7)$ & $8(4.2)$ & $1(0.7)$ & $-83.3 \%$ & \\
\hline & Total & $333(100.0)$ & $189(100.0)$ & $144(100.0)$ & - & \\
\hline
\end{tabular}

AR: acquired resistance; MDR: multidrug-resistant; XDR: extensively drug-resistant; PDR: pan drug-resistant.

$[12-14,23,24]$. Age distribution of providing patients $(74.5 \%$ adults and $21.1 \%<5$ years) differed from the national one, which shows less adults (53.9\%) and more under 5 children $(30.5 \%)$ [25]. The high frequency of female genital swabs in our sample could partly explain this gap. The predominance of enterobacteria (and especially E. coli) in our sample is consistent with the human bacterial pathogen's distribution.

The very high prevalence of AR was predictable. This high prevalence is now well documented and is one of the reasons for global mobilization on AMR. Interestingly, bacteria who scored a $100 \%$ AR rate in 2010 and 2017 are among the most frequent human pathogens (Enterobacteriaceae and other GNB). This also makes them the most exposed to antibiotic pressure (including inadequate use), which is thought to be involved in resistance occurrence [26].

In our knowledge, few has been published on class $1 \mathrm{WHO}$ priority resistance (especially Imipenem-R A baumannii, Imipenem-R $P$ aeruginosa, Imipenem-R Enterobacteriaceae) in Africa. However, recent studies reported imipenem-R Pseudomonas aeruginosa (Ipm- $\mathrm{R} \mathrm{Pa}$ ) rate of $11 \%$ in Nigeria [27] and $33 \%$ in Uganda [28], while we had $23.5 \%$ in 2017. An older South African study found a $42 \%$ Ipm-R Pa rate in 2007 [29], quite higher than what we got. Comparable disparities were observed for Imp-R Acinetobacter baumannii. Such disparities may be related to several factors such as drug policies, carbapenem availability, or financial access. These SSA rates of Imp-R non fermenting GNB (NF GNB) were quite higher than those observed in Western Europe in 2017-2018, which were $<10 \%$ [30-32]. However, they were still lower than the Iranian 54\% [33] and the 66 to $90 \%$ range found in Latin America [34]. The rapid increase of Imp-R NF GNB in Cameroon should lead to caution in antibiotic use, since there is no alternative to face highly resistant bacteria in the country.

Carbapenem-R Enterobacteriaceae appeared to have a low and stable frequency in our study. Higher values were found in Morocco [35]. Our 3GC-R Enterobacteriaceae prevalence was lower than those found in earlier Cameroonian studies, around $50 \%[12-14,23,36]$, probably due to sampling disparities. Since our enrollment was more global (including outpatients and hospitalized ones) than the one in those studies (hospitalized patients only). We found no study on Vancomycin-R enterococcus in Africa, which appeared to be emerging in Cameroon. This may cause serious therapeutic issues. Methicillin-R Staphylococcus aureus (MRSA) was more frequent in our study than in other SSA recent studies, where its prevalence ranged from $0 \%$ in Zambia to $7 \%$ in Zimbabwe [37-39]. This high prevalence may compromise patient management, since the Cameroonian minimum wage, 52.3 euros [40], cannot afford a daily dose of vancomycin (unique anti-MRSA available in Cameroon). Our FQ-R Campylobacter prevalence was also high compared to other SSA series, ranging from 20 to $33 \%[41,42]$. 
Meanwhile, FQ-R Salmonella in 2010 close to $4.3 \%$ found one year later in Congo [43]. The seven-year increase $(+142 \%)$ of the latter is easily explained by a very anarchical and overuse of FQ in our country. This overuse could also explain the very high frequency of FQ-R Neisseria gonorrhoeae. Ampicillin-R $H$. influenzae showed very high values, compared to 8.7$46 \%$ range found in other countries $[35,44,45]$.

The formula used to identify MDR could explain discrepancies with related specific resistances (MRSA and 3GC-R Enterobacteriaceae for example), since it included several antibiotics rather than specific ones. MDR/XDR Acinetobacter spp prevalence was consistent with the worldwide survey led by Lob in 2016; it ranged from 47\% in Northern America to $93 \%$ in Middle East [46]. We found no study from Africa using the same definition of MDR and XDR for other eligible pathogens. A local comparison could therefore not be made.

Our study design did not permit to assess what happened within the 2010-2017 interval. Therefore, real trends could be different from what our results assumed. The retrospective enrollment led to a huge number of excluded specimen and missing data, increasing risk of information bias. Data on providing patients were insufficient, making clinical correlation impossible and population profile nonassessable. However, the accessibility and affordability of CPC allow inclusion of patients from different regions and social categories. Every citizen can realize tests in CPC, and discounts up to $50 \%$ are applied for students, children, the elderly, civilian servants, and hospitalized patients from public hospitals. Whole-year enrollment was an advantage since it allowed to overcome potential between-season variations in circulating pathogens. This study succeeded in informing one of the WHO action plan's objectives, especially with the assessment of $\mathrm{WHO}$ priority resistances. Using data from a reference laboratory with cutting-edge equipment was a quality guarantee.

Taking into consideration, these AMR's prevalence may help Cameroonian clinicians in their daily practice, given that very few health facilities all around the country can attend a proper bacteriologic test. High prevalence of serious resistances could encourage decision-makers to build programs addressing AMR in the country. They could also be used to sensitize health workers and medical students on the reality of this phenomenon in the country and support training on this important issue.

Clinical data related to identify bacteria could not be reached. Prospective designs should overcome this limitation. The real dynamic of AMR evolution could not be assessed in this study, but trends have been revealed. Repeated similar studies or cohorts would be needed to have a long-term evolution. Extrapolation of these results to the whole country should be cautious, and local or regional studies would be necessary to complete them. Circulating bacteria are not limited to humans and patients; environment and animals must be studied as well.

\section{Conclusion}

In this study designed to assess AMR trends in Cameroon, most of bacteria of clinical interest have shown high resis- tance rates, which increased between 2010 and 2017, especially WHO priority pathogens. Almost all the strains identified had an acquired resistance. The prevalence of MDR was high, while XDR remained low but increased, and PDR was absent. These results contributed to improve knowledge on AMR in Cameroon and advocate for an urgent need of public health strategies against this threat. There is a need for regular surveillance of antibacterial resistance to keep decision-makers and clinicians informed.

\section{Data Availability}

The authors of the present manuscript did not received special access privileges to the data which belong to the Centre Pasteur Cameroon, a public institution of the Ministry of public health. Most of them work at Centre Pasteur du Cameroun. To get access to the study data; a researcher should submit a request detailing the planned analysis on the requested data. Requests should be sent to the CPC (cpc@pasteur-yaounde.org) or to Dr. Tejiokem Mathurin (tejiokem@pasteur-yaounde.org). All requests will be reviewed by the scientific department.

\section{Disclosure}

It was performed as part of the Public Health training of the corresponding author, from Bordeaux school of public health. It was performed as part of employment of the coauthors, who worked both in Centre Pasteur Cameroon and University of Bordeaux.

\section{Conflicts of Interest}

The authors declare no conflict of interest for this study.

\section{Acknowledgments}

The authors acknowledge both staff of bacteriology unit and epidemiology unit of Centre Pasteur du Cameroun.

\section{References}

[1] OMS, Résistance aux antimicrobiensApril 2018, http://www .who.int/mediacentre/factsheets/fs194/fr/.

[2] World Health Organisation, Worldwide country situation anal$y$ sis :: response to antimicrobial resistance, 2015, April 2018 http://apps.who.int/iris/bitstream/handle/10665/163468/ 9789241564946_eng.pdf;jsessionid=A11C214722F6E3D8E519 D1B88E399B0C? sequence=1.

[3] H. C. Wegener, "Antibiotics in animal feed and their role in resistance development," Current Opinion in Microbiology, vol. 6, no. 5, pp. 439-445, 2003.

[4] T. W. Alexander, L. J. Yanke, E. Topp et al., "Effect of subtherapeutic administration of antibiotics on the prevalence of antibiotic-resistant Escherichia coli bacteria in feedlot cattle," Applied and Environmental Microbiology, vol. 74, no. 14, pp. 4405-4416, 2008.

[5] A. R. Vieira, P. Collignon, F. M. Aarestrup et al., "Association between antimicrobial resistance in Escherichia coli isolates from food animals and blood stream isolates from humans 
in Europe: an ecological study," Foodborne Pathogens and Disease, vol. 8, no. 12, pp. 1295-1301, 2011.

[6] I. Khattak, M. H. Mushtaq, S. Ayaz et al., "Incidence and drug resistance of zoonotic Mycobacterium bovis infection in Peshawar, Pakistan," Advances in Experimental Medicine and Biology, vol. 1057, pp. 111-126, 2018.

[7] F. M. Aarestrup, "Veterinary drug usage and antimicrobial resistance in bacteria of animal origin," Basic \& Clinical Pharmacology \& Toxicology, vol. 96, no. 4, pp. 271-281, 2005.

[8] S. Schwarz, C. Kehrenberg, and T. R. Walsh, "Use of antimicrobial agents in veterinary medicine and food animal production," International Journal of Antimicrobial Agents, vol. 17, no. 6, pp. 431-437, 2001.

[9] J. A. Ayukekbong, M. Ntemgwa, and A. N. Atabe, “The threat of antimicrobial resistance in developing countries: causes and control strategies," Antimicrobial Resistance and Infection Control, vol. 6, no. 1, p. 47, 2017.

[10] S. Ur Rahman and M. Mohsin, "The under reported issue of antibiotic-resistance in food-producing animals in Pakistan," Pakistan Veterinary Journal, vol. 39, pp. 2074-7764, 2019.

[11] WHO, Antimicrobial resistance: global report on surveillance 2014April 2018, http://www.who.int/drugresistance/ documents/surveillancereport/en/.

[12] J.-F. T. K. Akoachere, S. Yvonne, N. H. Akum, and E. N. Seraphine, "Etiologic profile and antimicrobial susceptibility of community-acquired urinary tract infection in two Cameroonian towns," BMC Research Notes, vol. 5, no. 1, p. 219, 2012.

[13] J. Gangoue-Pieboji, S. Koulla-Shiro, P. Ngassam, D. Adiogo, and P. Ndumbe, "Antimicrobial activity against gram negative bacilli from Yaounde Central Hospital, Cameroon," African Health Sciences, vol. 6, no. 4, pp. 232-235, 2006.

[14] R. N. Ndip, E. A. Ntiege, L. M. Ndip, G. Nkwelang, J. F. T. K. Akoachere, and T. N. Akenji, "Antimicrobial resistance of bacterial agents of the upper respiratory tract of school children in Buea, Cameroon," Journal of Health, Population and Nutrition, vol. 26, no. 4, pp. 397-404, 2008.

[15] C. M. Lonchel, P. Melin, J. Gangoué-Piéboji, M.-C. O. Assoumou, R. Boreux, and P. De Mol, "Extended-spectrum $\beta$-lactamase-producing Enterobacteriaceae in Cameroonian hospitals," European Journal of Clinical Microbiology \& Infectious Diseases, vol. 32, no. 1, pp. 79-87, 2013.

[16] WHO, Global action plan on antimicrobial resistanceApril 2020, http://www.who.int/antimicrobial-resistance/ publications/global-action-plan/en/.

[17] Société française de Microbiologie, Référentiel en microniologie, p. 370, Société française de Microbiologie, 4 edition, 2010.

[18] Société française de Microbiologie, Référentiel en Microbiologie, p. 854, Société française de Microbiologie, 5 edition, 2015.

[19] Société Française de Microbiologiecasfm_2010.pdf, April2018, http://www.sfm-microbiologie.org/UserFiles/files/casfm_2010 .pdf.

[20] SFM - EUCAST, Comité de l'antibiogramme de la Société Française de MicrobiologieRecommandations 2016, April 2018, http://www.sfm-microbiologie.org/UserFiles/files/casfm/ CASFM2016_V1_0_FEVRIER.pdf.

[21] WHO, WHO publishes list of bacteria for which new antibiotics are urgently neededApril 2020, https://www.who.int/newsroom/detail/27-02-2017-who-publishes-list-of-bacteria-forwhich-new-antibiotics-are-urgently-needed.

[22] A.-P. Magiorakos, A. Srinivasan, R. B. Carey et al., "Multidrugresistant, extensively drug-resistant and pandrug-resistant bacteria: an international expert proposal for interim standard definitions for acquired resistance," Clinical Microbiology and Infection, vol. 18, no. 3, pp. 268-281, 2012.

[23] J. Gangoué-Piéboji, B. Bedenic, S. Koulla-Shiro et al., "Extended-spectrum-beta-lactamase-producing Enterobacteriaceae in Yaounde, Cameroon," Journal of Clinical Microbiology, vol. 43, no. 7, pp. 3273-3277, 2005.

[24] N. Agyepong, U. Govinden, A. Owusu-Ofori, and S. Y. Essack, "Multidrug-resistant gram-negative bacterial infections in a teaching hospital in Ghana," Antimicrobial Resistance and Infection Control, vol. 7, no. 1, p. 37, 2018.

[25] BUCREP, Projections démographiquesMay 2018, http://www .bucrep.cm/index.php/fr/ressources-et-documentations/ telechargement/category/82-projections-demographiques.

[26] J.-P. Flandrois, "Evolution de la Résistance," in Bactériologie Médicale, pp. 97-100, Presses Universitaires de Lyon, Lyon, 1997.

[27] K. O. Zubair and K. C. Iregbu, "Resistance pattern and detection of metallo-beta-lactamase genes in clinical isolates of Pseudomonas aeruginosa in a central Nigeria tertiary hospital," Nigerian Journal of Clinical Practice, vol. 21, no. 2, pp. 176-182, 2018.

[28] D. P. Kateete, R. Nakanjako, M. Okee, M. L. Joloba, and C. F. Najjuka, "Genotypic diversity among multidrug resistant Pseudomonas aeruginosa and Acinetobacter species at Mulago Hospital in Kampala, Uganda," BMC Research Notes, vol. 10, no. 1, p. 284, 2017.

[29] A. Brink, J. Moolman, M. C. da Silva, and M. Botha, "Antimicrobial susceptibility profile of selected bacteraemic pathogens from private institutions in South Africa," South African Medical Journal, vol. 97, no. 4, pp. 273-279, 2007.

[30] A. Ramette, A. Kronenberg, and the Swiss Centre for Antibiotic Resistance (ANRESIS), "Prevalence of carbapenemresistant Acinetobacter baumannii from 2005 to 2016 in Switzerland," BMC Infectious Diseases, vol. 18, no. 1, p. 159, 2018.

[31] G. F. Schiavano, E. Carloni, F. Andreoni et al., "Prevalence and antibiotic resistance of Pseudomonas aeruginosa in water samples in central Italy and molecular characterization of oprD in imipenem resistant isolates," PLoS One, vol. 12, no. 12, article e0189172, 2017.

[32] N. Woodford, L. Xu-McCrae, S. Mushtaq et al., "Prevalence of carbapenem resistance and carbapenemase production among Enterobacteriaceae isolated from urine in the UK: results of the UK infection-Carbapenem Resistance Evaluation Surveillance Trial (iCREST-UK)," The Journal of Antimicrobial Chemotherapy, vol. 73, no. 3, pp. 698-702, 2018.

[33] H. Vaez, A. Salehi-Abargouei, and F. Khademi, "Systematic review and meta-analysis of imipenem-resistant Pseudomonas aeruginosa prevalence in Iran," Germs, vol. 7, no. 2, pp. 86-97, 2017.

[34] J. A. Labarca, M. J. C. Salles, C. Seas, and M. Guzmán-Blanco, "Carbapenem resistance in Pseudomonas aeruginosa and Acinetobacter baumannii in the nosocomial setting in Latin America," Critical Reviews in Microbiology, vol. 42, no. 2, pp. 276-292, 2016.

[35] Société Marocaine d'infectiologie pédiatrique, Le-guide-pratique-des-bacteries-pathogenes. pdf, 2017, May 2018, https:// pharmacie.ma/uploads/pdfs/Le-guide-pratique-des-bacteriespathogenes.pdf.

[36] C. L. Magoué, P. Melin, J. Gangoué-Piéboji, M.-C. Okomo Assoumou, R. Boreux, and P. De Mol, "Prevalence and spread 
of extended-spectrum $\beta$-lactamase-producing Enterobacteriaceae in Ngaoundere, Cameroon," Clinical Microbiology and Infection, vol. 19, no. 9, pp. E416-E420, 2013.

[37] L. Ampaire, A. Muhindo, P. Orikiriza, J. Mwanga-Amumpaire, L. Bebell, and Y. Boum, "A review of antimicrobial resistance in East Africa," Afr J Lab Med., vol. 5, no. 1, p. 432, 2016.

[38] K. Mauchaza, F. D. Madzimbamuto, and S. Waner, "Methicillin-resistant Staphylococcus aureus in Zimbabwe," Ghana Medical Journal, vol. 50, no. 2, pp. 68-71, 2016.

[39] M. M. B. Nagelkerke, K. Sikwewa, D. Makowa, I. de Vries, S. Chisi, and J. W. Dorigo-Zetsma, "Prevalence of antimicrobial drug resistant bacteria carried by in- and outpatients attending a secondary care hospital in Zambia," BMC Research Notes, vol. 10, no. 1, p. 378, 2017.

[40] Premier Ministre Cameroun, Décret portant revalorisation du Salaire Minimum Interprofesionnel Garanti (SMIG). 2014/2217, p. 1, 2014, http://www.spm.gov.pm.

[41] E. R. Vlieghe, J. A. Jacobs, M. Van Esbroeck, O. Koole, and A. Van Gompel, "Trends of norfloxacin and erythromycin resistance of Campylobacter jejuni/Campylobacter coli isolates recovered from international travelers, 1994 to 2006," Journal of Travel Medicine, vol. 15, no. 6, pp. 419-425, 2008.

[42] C. O. Shobo, L. A. Bester, S. Baijnath, A. M. Somboro, A. K. C. Peer, and S. Y. Essack, "Antibiotic resistance profiles of Campylobacter species in the South Africa private health care sector," Journal of Infection in Developing Countries, vol. 10, no. 11, pp. 1214-1221, 2016.

[43] O. Lunguya, V. Lejon, M.-F. Phoba et al., "Antimicrobial resistance in invasive non-typhoid Salmonella from the Democratic Republic of the Congo: emergence of decreased fluoroquinolone susceptibility and extended-spectrum beta lactamases," PLoS Neglected Tropical Diseases, vol. 7, no. 3, article e2103, 2013.

[44] D. F. Sahm, N. P. Brown, C. Thornsberry, and M. E. Jones, "Antimicrobial susceptibility profiles among common respiratory tract pathogens: a GLOBAL perspective," Postgraduate Medicine, vol. 120, no. 3, Supplement 1, pp. 16-24, 2008.

[45] I. Mandomando, M. Espasa, T. Nhampossa et al., "Antimicrobial drug resistance trends of bacteremia isolates in a rural hospital in southern Mozambique," The American Journal of Tropical Medicine and Hygiene, vol. 83, no. 1, pp. 152-157, 2010.

[46] S. H. Lob, D. J. Hoban, D. F. Sahm, and R. E. Badal, "Regional differences and trends in antimicrobial susceptibility of Acinetobacter baumannii," International Journal of Antimicrobial Agents, vol. 47, no. 4, pp. 317-323, 2016. 